Annuaire suisse de politique de développement

22-2 | 2003

Société de l'information et coopération internationale

\title{
Droits d'auteur : vers une nouvelle forme de colonialisme
}

Brian Wafawarowa

\section{(2) OpenEdition}

1 Journals

Édition électronique

URL : http://journals.openedition.org/aspd/583

DOI : 10.4000/aspd.583

ISSN : 1663-9669

Éditeur

Institut de hautes études internationales et du développement

Édition imprimée

Date de publication : 1 novembre 2003

Pagination : 185-188

ISSN : 1660-5934

\section{Référence électronique}

Brian Wafawarowa, "Droits d'auteur : vers une nouvelle forme de colonialisme », Annuaire suisse de politique de développement [En ligne], 22-2 | 2003, mis en ligne le 23 mars 2010, consulté le 08

septembre 2020. URL : http://journals.openedition.org/aspd/583 ; DOI : https://doi.org/10.4000/aspd. 583

(c) The Graduate Institute | Geneva 


\title{
Droits d'auteur: vers une nouvelle forme de colonialisme
}

\author{
Brian Wafawarowa*
}

1 est urgent pour le monde en développement d'améliorer son accès à l'information, particulièrement dans les domaines cruciaux que sont l'éducation, la médecine, le droit et les droits de l'homme. Pour y parvenir, nous devons disposer d'un secteur de l'information viable, capable de créer, publier et diffuser des contenus pertinents. Un bon équilibre entre accès à l'information et viabilité du secteur de l'information est essentiel.

Le manque d'accès découle entre autres de la pauvreté, du prix élevé des livres et du matériel technique ainsi que des coûts de distribution. Les prix découlent de la quasi absence d'économies d'échelle, du piratage et des copies illégales.

La majeure partie des livres sur l'accès à l'information au Sud sont produits au Nord. Selon de récentes recherches de l'Association pour le développement de l'éducation en Afrique (ADEA) et du Réseau des éditeurs africains (APNET), l'Afrique consomme environ $12 \%$ de tous les livres lus dans le monde, mais en produit moins de $3 \%^{1}$. C'est pourquoi les campagnes qui visent à améliorer l'accès à l'information sans rappeler que le monde en développement doit participer activement à la création et la diffusion de l'information sont inadéquates.

Comme ce fut le cas de la révolution de l'imprimerie, la révolution digitale offre au monde un nouveau bond technologique mais laissera une grande partie de la population sur la touche, à moins que les stratégies proposées aujourd'hui prennent également en compte les problèmes du monde en développement en matière de matériel et de taux de raccordement. En Afrique, ce taux est d'environ $7 \%$, soit bien inférieur aux $25 \%$ nécessaires à la dissémination durable de l'information électronique ${ }^{2}$.

Dans cette perspective, l'une des principales questions est celle des «nouvelles exceptions légales (NEL) à la protection des droits d'auteur», soit l'adoption de nouvelles limitations à son application.

Le droit d'auteur (ou copyright) est une forme de propriété intellectuelle protégée par la loi, en particulier par la Convention de Berne qui, en la matière, constitue le cœur d'un dispositif internationalement reconnu. Le droit d'auteur protège notamment les œuvres écrites et accorde à leur auteur un droit exclusif sur leur reproduction ou toute autre utilisa- 
tion, y compris sur l'Internet. Il est internationalement reconnu dans la Déclaration universelle des droits de l'homme (article 27[2]). Le droit d'auteur protège l'expression des idées, des opinions et des faits, mais non les idées, les opinions ou les faits eux-mêmes.

Les exceptions au droit d'auteur autorisent légalement la copie d'extraits d'une œuvre, mais à certaines fins uniquement, par exemple la citation, la critique ou l'information d'actualité. Elles permettent également l'adaptation des œuvres dans certains cas limités bien précis tels que la parodie ou le pastiche.

Dans la vive discussion actuelle sur le droit d'auteur, d'aucuns affirment que pour favoriser l'accès aux œuvres sous copyright, en particulier dans les pays en développement, il faut augmenter le nombre de ces exceptions. Leurs arguments sont doublement problématiques. Tout d'abord, ils ignorent fréquemment le rôle des créateurs du Sud, ne retenant que les utilisateurs; cette erreur de perspective a des parfums de néocolonialisme. Ensuite, ils oublient que les créateurs et éditeurs du Sud ont besoin d'incitations spéciales pour poursuivre leurs efforts et investir dans une information produite par le Sud. Qu'on l'ignore, et le Sud restera un consommateur plutôt qu'un créateur d'information.

En fait, les NEL ne prennent pas en compte la chaîne de l'information et son fonctionnement. Les stratégies de diffusion de l'information qui ignorent sa création ont peu de chance de succès. Les détenteurs de droits doivent travailler dans un cadre réglementé où les investissements engagés dans la publication et la diffusion offrent des perspectives de rentabilité. En Afrique, ce n'est pas actuellement le cas du secteur du livre et de l'information. Les diffuseurs ont besoin de matériel en rapport avec les réalités locales. Une stratégie ambitieuse d'accès à l'information dans le monde en développement doit donc chercher à créer les conditions qui favorisent la créativité plutôt que de l'étouffer.

L'adoption des NEL dans le monde en développement ne fera qu'éroder la compétitivité de son secteur du livre et de l'information, perpétuant un ordre néocolonial dans lequel notre regard sur le monde et sur nous-mêmes sera marqué du sceau colonial puisque seuls les détenteurs de droits également actifs dans les parties plus solvables du monde seront en mesure de distribuer l'information dans le monde en développement. Inutile de souligner l'impact d'une telle situation sur la qualité de l'éducation.

Le monde en développement n'a pas uniquement besoin d'accéder à l'information, il a besoin d'informations pertinentes et appropriées. Il a besoin de participer sur un pied d'égalité au système global de l'information. Pour y parvenir, il doit être à la fois consommateur et producteur de contenu global. Vouloir ignorer ces questions - quel type d'information? Produite par qui ? Destinées à qui ? ne fera que perpétuer l'hégémonie actuelle du secteur de la diffusion.

Les sujets proprement africains traités par des étrangers (et dépassant le regrettable «regard africain» fasciné par les mutilations génitales et la sorcellerie) rencontrent un intérêt croissant. Il serait naturel que des auteurs 
et éditeurs africains exploitent aussi cette veine, au profit du secteur africain de l'information. Mais il est très probable que l'adoption des NEL les en empêche. Pour que la protection du savoir indigène nous profite aussi, il est indispensable que les rapports de concurrence avec le reste du monde soient équitables. Si ce n'est pas le cas, si notre capacité d'exploiter ces contenus reste compromise, les auteurs, les érudits et les éditeurs craignent que nous en soyons dépossédés. Chido Nwangwu, d'USAfrica Online, pense que «si on ne la surveille pas attentivement, la révolution digitale risque de s'avérer un outil d'impérialisme encore plus brutal que le colonialisme $»^{3}$.

L'application des NEL doit être mise en cause jusque dans les domaines où les besoins sont les plus criants, comme dans l'éducation. L'effet de telles exceptions dans le cadre africain où $95 \%$ des livres sont des livres scolaires, contre $35 \%$ seulement dans le monde développé ${ }^{4}$, demande un sérieux examen. Le secteur scolaire subventionne d'autres types de publications. Cependant, les conditions économiques qui y prévalent sont si précaires que l'espoir des éditeurs est de produire des livres moins coûteux que leur photocopie, puis de s'y retrouver sur la quantité. Aujourd'hui, dans tous les pays d'Afrique, les départements de l'éducation et les éditeurs cherchent ensemble comment produire du matériel de formation accessible. C'est pourquoi, en l'occurrence, l'image de la grande entreprise exploitant les masses appauvries grâce aux droits d'auteur ne s'applique pas.
L'application des NEL porte en elle une contradiction. On discute d'une part de «plate-forme traditionnelle amorphe», sans structure, dans le monde digital, à travers le world wide $w e b$, et, d'autre part, on suppose que ce monde peut encore être divisé et géré comme le monde en développement avec les NEL pendant que le monde développé dispose d'un jeu de règles différent. Cette contradiction soulève une question: comment ces exceptions seront-elles mises en œuvre et gérées?

Notre unique chance de survivre dans le monde en développement en tant que détenteurs de droits et diffuseurs d'information est d'engager le dialogue entre les détenteurs de droits, leurs utilisateurs, les gouvernements, les professionnels de l'information et les agences de développement. Le fait que le symposium sur le copyright de l'Union internationale des éditeurs se soit tenu au Ghana en 2002 démontre que le droit d'auteur est aussi important pour le monde en développement que pour le monde développé. Comme nous l'avons souligné dans plusieurs contributions présentés à la Conférence Infoethics de l'UNESCO à Paris en 2000, les problèmes de capacité du monde en développement doivent être résolus par le renforcement de cette capacité, la formation et l'application de lois effectives sur le développement du secteur de l'information et sur l'amélioration de l'accès à l'information, et non pas par des solutions ciblées sur un seul aspect du secteur de l'information au détriment de sa compétitivité globale. 
En conclusion, les solutions doivent tenir compte des circonstances réelles du monde en développement. Elles doivent procéder d'un large désir d'encourager le développement holistique du secteur de l'information. Il est également important d'évaluer l'impact de toute solution sur la participation locale et les diverses contributions globales au savoir. Nous devons réfléchir aux moyens de subventionner l'accès à l'information plutôt que d'accabler les détenteurs de droits. Le secteur privé local n'est pas en mesure de le faire seul. Si l'accès universel à l'information est une ambition plausible pour le monde en développement, il incombe à la société - gouvernement et secteur privé compris - de l'assurer et de l'améliorer.

\section{Références}

African Publishing Review, September 1998.

African Publishing Review, October 2000.

Askerud P., A Guide to Sustainable Book Development, Paris, UNESCO, 1997.

Brass D., The Future of Publishing, 26th IPA Congress, 2000.

Makhotsi R., Promoting Intra-African Book Trade, APNET and ADEA, 1999.

Wafawarowa B., Free Flow of Information, 26th IPA Congress, 2000. 